\title{
Bildungsaspiration, Begabung und Schullaufbahn: Eltern als Erfolgspromotoren?
}

\section{Margrit Stamm}

Der Aufsatz untersucht den Zusammenhang von elterlichen Bildungsaspirationen und Schulkarrieren der Söhne und Töchter. Als empirische Basis dient eine Längsschnittuntersuchung von 400 heute 17jährigen Jugendlichen zu den Wirkungen des vorschulischen Kompetenzerwerbs in Lesen und Mathematik. Untersucht wurde, inwiefern sich Bildungsaspirationen von Eltern im Schulerfolg ihres Kindes und seinen Berufsambitionen am Ende der obligatorischen Schulzeit niederschlagen und ob dies für Familien mit überdurchschnittlich begabten Kindern im Besonderen zutrifft. Grundsätzlich legen die Analysen eine Unterscheidung von fünf Grundmustern elterlicher Bildungsaspiration nahe. Zwei Muster erweisen sich dabei als bedeutsam hinsichtlich der Differenzierung nach hohen Begabungsprofilen. Es sind dies (a) eine «bildungsgewohnte» und -ambitionierte Elterngruppe, deren Kind sowohl eine erfolgreiche Schullaufbahn hinter sich als auch vergleichbare Berufsambitionen entwickelt hat; (b) eine Elterngruppe mit polaren Tendenzen, genannt "die Fordernden", weil sich ihre Bildungsaspirationen vor allem in einer hohen Anspruchsorientierung manifestieren, ihr Kind jedoch auf eine eher misserfolgreiche Schulkarriere zurückblickt und sich seine beruflichen Ambitionen nicht in den Vorstellungen der Eltern spiegeln.

\section{Problematik}

Gemäss den kantonalen Schulgesetzen ist die Erziehung der heranwachsenden Generation in der Schweiz gemeinsame Aufgabe von Schule und Elternhaus. De facto ist sie allerdings vielerorts nur beabsichtigt, kaum jedoch realisiert. Dies war ein wesentlicher Befund des Jahreskongresses 2003 ,Schule und Familie Perspektiven einer Differenz' der Schweizerischen Gesellschaft für Bildungsforschung in Bern (Neuenschwander, 2004; Neuenschwander, Balmer, Gasser, Golz, Hirt, et al. 2005). Dieser Befund repliziert damit nicht nur aus den frühen neunziger Jahren stammende Erkenntnisse (Moser, 1992; Stöckli, 1992), sondern bildet zugleich das theoretische Argumentarium des Dachverbandes Lehre- 
rinnen und Lehrer Schweiz, der in der Zusammenarbeit zwischen Schule und Elternhaus noch grosse Mängel ortet (Dachverband Lehrerinnen und Lehrer Schweiz, 2004). Ähnlich sieht es im deutschsprachigen Ausland aus, wo Krumm (1996) oder Wild \& Hofer (2002) die gegenwärtig praktizierten Formen der Eltern-Lehrer-Kooperation als unzureichend und ineffektiv kommentieren. Dass Elternarbeit häufig zum Randbereich der Tätigkeit von Lehrpersonen gehört (Pekrun, 2001), wird mit Defiziten in der Lehrerbildung (Krumm, 1996) mit nicht honorierter Mehrarbeit oder mit egozentrischen Elterninteressen (Rolff, Bauer, Klemm \& Pfeiffer, 1996) begründet. In den USA hat der manifeste Beratungsbedarf der Eltern inzwischen zur Entwicklung von Programmen geführt, die Eltern und Lehrpersonen in einer wirksameren Zusammenarbeit unterstützen sollen. Erste Evaluationen belegen, dass bereits regelmässig durchgeführte Informationsabende eine intensivere Eltern-Lehrerkooperation zur Folge haben (Cooper, Lindsay \& Nye, 2000).

Nimmt man allerdings Untersuchungen zur Kenntnis, wonach elterliche Betreuung in Form von Hausaufgabenhilfe oder Finanzierung von Nachhilfe weit verbreitet ist, so müsste man zumindest von einer gut etablierten, aber ,heimlichen' Zusammenarbeit ausgehen (Krumm, 1996; Hampsch, 1998). Deshalb erstaunt zwar, dass dieser Sachverhalt bislang kaum näher untersucht ist, doch kann die Ursache nicht zuletzt in der Pädagogik selbst vermutet werden, die lange Jahre aufgrund ihres engen Fokus auf die Schule andere Lernorte mehrheitlich ausgeblendet hat. Diese Situation ist denn auch verschiedentlich kritisiert (vgl. zusammenfassend: Pekrun, 1997) und mit einer verstärkten Ausrichtung auf familienorientierte Kontexte im Sinne des ökologischen Ansatzes von Bronfenbrenner (1981) beantwortet worden. So wird vor dem Hintergrund des sozialen Wandels, zu dessen Kernpunkten die Emotionalisierung der Kindheit und die Bedeutungszunahme des Bildungskapitals gehören, verständlich, dass der Familienalltag zunehmend von, verschulten' Eltern-Kind-Beziehungen geprägt ist (Ulich, 1989; Stöckli, 1992). Von besonderem Interesse sind deshalb die vereinzelt vorgelegten Abhandlungen zum «Bildungsehrgeiz der Eltern» (Zinnecker \& Georg, 1996; Stecher, 1999). Ausgeblendet geblieben sind in diesen Studien jedoch mögliche Zusammenhänge von Begabung und Bildungsaspiration. Erinnert sei dabei an Alice Millers «Drama des begabten Kindes» (1980), welches das Augenmerk gerade auf solche Zusammenhänge und damit auf Kinder richtet, die sich den Wünschen und Bedürfnissen der Eltern anpassen, möglicherweise auch gegen den eigenen Willen. Wenngleich der Aussagengehalt der Publikation Millers wissenschaftlich umstritten blieb, so macht sie doch auf mögliche $\mathrm{Zu}$ sammenhänge von Begabung und Bildungsaspiration aufmerksam. Auch aus der Hochbegabungsforschung liegen vereinzelt Hinweise vor, wonach Eltern angesichts des herausragenden Potenzials ihrer Kinder besonders ausgeprägte Erwartungsorientierungen aufbauen und sich bemühen, diese auf die Kinder zu übertragen. 
Der vorliegende Aufsatz versucht unter Zuhilfenahme von Daten einer Schweizer Längsschnittstudie solche Vermutungen zu konturieren. Zunächst werden einige theoretische Konzepte und empirische Studien zur Erklärung von Elternerwartungen und ihrer Abbildung in den Schullaufbahnen und Berufsambitionen ihrer Kinder dargestellt und darauf aufbauend die Hauptfragestellungen formuliert. Im Mittelpunkt stehen sodann die Ergebnisse der Studie, die anhand von vier Dimensionen präsentiert werden. Den Abschluss des Aufsatzes bildet schliesslich die Diskussion der Befunde.

\section{Bildungsaspiration und Schulerfolg aus theoretischer und empirischer sicht}

Die Frage elterlicher Einflussnahme auf die Schullaufbahn ihres Kindes wird von verschiedenen Disziplinen theoretisch bearbeitet. In diesem Aufsatz werden drei Konzepte herausgegriffen, die für die vorliegende Thematik besondere Relevanz aufweisen: die Kapitaltheorie nach Bourdieu (1983), handlungstheoretische Modelle zu Bedingungsfaktoren des Schulerfolgs sowie der Selbstbestimmungsansatz nach Deci \& Ryan (1993). Während die ersten beiden Ansätze versuchen, die Faktoren elterlicher Bildungsaspiration und deren unterschiedlichen Wirkungen zu erklären, versucht der dritte Ansatz aufzuzeigen, weshalb sich Kinder entsprechend den elterlichen Erwartungen verhalten und wann diese erfolgreich oder misserfolgreich sein können.

Zwar ist die Erkenntnis, dass eine an Bildung und Leistung orientierte Statuserwerbsphilosophie der Eltern den Schulerfolg fördert, ein Fakt des Colemanund des Plowden-Reports (Coleman, Campbell, Hobson, McPartland, Mood, Weinfeld \& York, 1966; Plowden, 1967), der nachfolgend in vielen Untersuchungen bestätigt worden ist (vgl. zusammenfassend Fraser, Walberg, Welch \& Hattie, 1987). Doch hat im deutschsprachigen Raum erst Bourdieus Kapitaltheorie zu differenzierteren Überlegungen geführt, wonach das ökonomische, das soziale und das kulturelle Kapital drei Dimensionen darstellen, welche die elterlichen Bildungsambitionen determinieren. Während das ökonomische Kapital darüber bestimmt, welche Ressourcen den Kindern zur Verfügung stehen, umfasst das kulturelle (intellektuelle) Kapital alle Einstellungen der Familie, ihren Bildungsgrad, ihr Wissen, ihre Fähigkeiten, aber auch ihre Erwartungsorientierungen. Dementsprechend müssten in bildungsnahen Sozialschichten am deutlichsten ausgeprägte Bildungsaspirationen erwartet werden. Handlungstheoretische Modelle hingegen sprechen weniger dem Status der Eltern primäre Bedeutung zu als der innerfamiliären Beeinflussung. In dieser Sichtweise werden elterliche Erwartungsorientierungen und die Leistungen der Kinder sowohl zur Ursache als auch zur Folge der Leistungsfähigkeit. Ausschlaggebend ist gemäss Holodynski \& Oerter (2002, S. 551ff.), dass sich Erfolgserwartungen der Eltern bei der Ausbildung des Anspruchsniveaus modellhaft auf ihr Kind übertragen 
und sich bei ihm als hohe Erfolgserwartung bei niedriger Misserfolgsängstlichkeit artikulieren. Anders hingegen der Selbstbestimmungsansatz von Deci \& Ryan (1993). Er erklärt das Zustandekommen intrinsischer und die erleichterte Integration extrinsischer Motivation über die elterliche Förderung von Grundbedürfnissen wie Autonomie, Kompetenzerleben und soziale Einbindung. Aufbauend auf den Kernannahmen dieser Theorie untersucht Wild (2001) den Zusammenhang familialer und schulischer Bedingungen der Lernmotivation.

Mit Blick auf die Hochbegabungsforschung ist davon auszugehen, dass sich Erwartungsorientierungen von Eltern überdurchschnittlich begabter Kinder ebenfalls solchen Ansätzen zuordnen lassen. Entsprechend liesse sich argumentieren, dass die Erwartungen der Eltern durch früh artikulierte Kompetenzen des Kindes (beispielsweise vorschulisches Lesen und Rechnen, grosser Wissensdrang oder hohe Kreativität) schon im Kleinkindalter erfüllt und im weiteren Entwicklungsverlauf derart stimuliert werden, dass die Bereitschaft zu Investitionen steigt. In der Tat finden sich verschiedene Studien, welche solche Zusammenhänge stützen. Stellvertretend seien Blooms (1985) oder Cornells (1984) Studien genannt, welche belegen, dass Eltern hoch begabter Kinder Schulleistungen im Allgemeinen positiv bewerten, hohe Bildungsabschlüsse erwarten und die Potenzialentfaltung ihres Nachwuchses mit hohen finanziellen Ressourcen fördern. Mit Blick auf die Underachievement-Forschung erweisen sich solche Argumentationen jedoch als widersprüchlich oder gar unzutreffend (vgl. zusammenfassend Whitmore, 1980; kritisch: Tettenborn, 1996). Trotz bildungsund leistungsorientierter Statuserwerbsphilosophie der Eltern gestalten sich die Leistungsentwicklungen ihrer Kinder eher erwartungswidrig, begleitet von deutlichen Anzeichen des Underachievements. Unter diesem Begriff werden Kinder und Jugendliche mit überdurchschnittlich ausgeprägtem Begabungspotenzial subsumiert, die nicht in der Lage sind, dieses in entsprechende Leistung zu transferieren, so dass ihre Leistungen über einen längeren Zeitraum deutlich schlechter ausfallen als die Leistungen des Klassendurchschnitts. Möglicherweise verbergen sich hinter den elterlichen Bildungsaspirationen spezifische Anspruchshaltungen, welche der Leistungsentwicklung abträglich sind und konflikthafte Schulkarrieren fördern (McCall, Evahn, \& Kratzer, 1992).

Fakt ist, dass sich aufgrund der verfügbaren empirischen Befunde die Annahme formulieren lässt, dass elterliche Bildungseinstellungen eine grössere Rolle spielen als alle anderen Variablen. Die Analysen solcher Einstellungsmuster konzentrieren sich dabei auf verschiedene Einflussmodelle, die sich in zwei grosse Bereiche einteilen lassen: in direkte Interventionen, welche auf das konkrete Verhalten der Kinder ausgerichtet sind und in indirekte, über Persönlichkeits- und Interessenentwicklung vermittelte Interventionen (vgl. Bierman, 1996). Direkte Interventionen manifestieren sich beispielsweise als Instruktion oder als Kontrolle von und Investition in häusliches Lernen. Gemäss Studien von Kramer \& Werner (1998), Hampsch (1998) oder der Deutschen Shell (2002) profitieren zwischen 25 Prozent und 35 Prozent der deutschen Kinder - 
vor allem aus gehobenen Sozialmilieus - von privater Nachhilfe. Indirekte Interventionen sind zum einen in den Einstellungsmustern der Eltern zu suchen. Empirische Beweise, wonach positive Schuleinstellungen der Eltern schulischen Erfolg ihrer Kinder beeinflussen, liefern Colemann (1988), Sauer \& Gamsjäger (1996) und Georg \& Zinnecker (1996). Je mehr die Eltern an Bildung und Leistung als Wege zum Berufserfolg glauben, desto mehr tun dies auch die Kinder. Diese Studien liefern damit empirischen Nachweise der kausalen Verbindung und Wirkung von Aspiration, Leistung und Schulverlauf. Indirekte Interventionen sind zum anderen auch in der Förderung intrinsischer Motivation zu suchen, wie sie von der Selbstbestimmungstheorie postuliert wird und in Untersuchungen von Krapp (1992) oder Allen, Moore, Kupermine und Bell (1998) nachgewiesen werden. Csikszentmihalyi \& Rathunde (1998) fokussieren dabei implizit auf überdurchschnittliche intellektuelle Begabung, wenn sie die optimale Förderung intrinsischer Motivation durch eine herausfordernde Passung an die individuellen Fähigkeiten des Kindes jenseits von Langeweile postulieren. Zu den indirekten Beeinflussungsgrössen gehören jedoch auch soziale UmweltKonfigurationen wie Peers oder andere, in der Freizeit erworbenen Leit- oder Vorbilder.

Bleibt noch die Frage zum Zusammenhang von Bildungsaspiration und sozialem Status. Entsprechende Studien bleiben in ihren Ergebnissen insgesamt widersprüchlich. Während Bourdieus (1992) oder Zinnecker \& Georgs (1996) Befunde positive Korrelationen zeigen, fallen Trudewind \& Windels (1991) oder Helmkes, Schrader, \& Lehneis-Klepper, (1991) Ergebnisse negativ aus. Des Weiteren belegt der kritische Überblick von Marjoribanks (1979) über Studien zu familiären Umwelten und ihre Wirkungen auf die kognitive Entwicklung der Kinder, dass schulische und berufliche Aspirationen der Eltern einen substantiellen Beitrag zur Vorhersage der Ambitionen ihrer Kinder leisten und dies auch für bildungsferne Milieus zutrifft. Zu ähnlichen Ergebnissen kommen Sauer \& Gamsjäger (1996) in ihrer gross angelegten Längsschnittstudie.

\section{Zusammenfassende Diskussion und Fragstellungen}

Was bedeuten die theoretischen Annahmen und die empirischen Ergebnisse für die vorliegende Fragestellung? Zunächst lassen die Befunde die Erkenntnis als gesichert erscheinen, dass sich die familiäre Umwelt einerseits als Funktion des sozialen, kulturellen und ökonomischen Kapitals und andererseits als ein, das kognitive Entwicklungsniveau und die Schulleistungen des Kindes unterschiedlich beeinflussendes Medium abbilden lässt. Deshalb ist anzunehmen, dass eine an Bildung und Leistung orientierte Statuserwerbsphilosophie der Eltern den Schulerfolg und auch das Bildungsverhalten der Kinder fördert. Allerdings ist, entsprechend den empirisch fundierten Annahmen der referierten Studien, zu erwarten, dass die Bildungsaspirationen der Eltern unterschiedlich ausgeprägt 
sind. Kaum gesichert sind hingegen Befunde zur Auswirkung der sozialen Herkunft auf Ausmass und Art der Bildungserwartungen, so dass offen bleiben muss, inwiefern die Wirkungen kulturellen, sozialen und ökonomischen Kapitals sozial disparat sind. Gleiches gilt für die familiären Umwelten Hochbegabter. Der Rückgriff auf die Hochbegabungsforschung legt zwar in der Tendenz ein überdurchschnittliches Bildungsengagement der Eltern nahe, doch verweisen die Underachievement-Befunde auf widersprüchliche Verhältnisse. Gänzlich unbeantwortet sind zudem Fragen zu den Wirkungen von elterlichen Bildungserwartungen, d.h. inwiefern sie sich in entsprechenden beruflichen Ambitionen ihrer Kinder niederschlagen.

Vor dem Hintergrund der skizzierten Befundlage sollen im Folgenden solche Problembereiche aufgegriffen und anhand des vorliegenden Datenmaterials diskutiert werden. Auf der Basis der referierten empirischen Befunde wird dabei eine kausale Verbindung und Wirkung von Aspiration, Leistung und Schulverlauf zwischen Eltern und Kind postuliert. Die familialen Bildungsaspirationen sollen jedoch nicht umfassend abgeklärt, sondern anhand einiger gezielter Fragestellungen diskutiert werden. Konkret handelt es sich um folgende Fragen:

(1) Wie artikulieren sich die Bildungsaspirationen der Eltern? Welche Unterschiede ergeben sich hinsichtlich sozialer Herkunft und kognitiven Fähigkeiten?

(2) In welchem Zusammenhang stehen Bildungsaspirationen von Eltern und Berufsambitionen ihres Kindes am Ende der obligatorischen Schulzeit? Welche familiären Aspirationstypen lassen sich herausfiltern?

(3) Ergeben sich Bezüge zwischen Bildungsaspirationen der Eltern, Berufsambitionen ihres Kindes und Schullaufbahn?

\section{Methode}

\section{Stichprobe}

Die Untersuchung stützt sich auf Daten aus der Längsschnittstudie «Frühlesen und Frührechnen als soziale Tatsachen». Die Stichprobe umfasst 366, heute 17jährige Jugendliche aus acht Schweizer Kantonen und dem Fürstentum Liechtenstein, die zur Hälfte einer Untersuchungsgruppe $(\mathrm{N}=185)$ und einer Vergleichsgruppe $(\mathrm{N}=181)$ einer ursprünglich 399 ProbandInnen umfassenden Stichprobe angehören. Der Untersuchungsgruppe zugeteilt wurde, wer im Herbst 1995, sechs Wochen nach Schuleintritt, in den über einen standardisierten Test erhobenen Aufgaben in Lesen und Mathematik fehlerfrei gearbeitet hatte. Jedem dieser Kinder wurde ein Vergleichsgruppenkind gleichen Geschlechts gegenübergestellt, das über keine entsprechenden Vorkenntnisse verfügte, jedoch die gleiche Klasse besuchte. $24 \%$ der Stichprobe (88 Jugendliche) verfügen über Intelligenzwerte $\geq 120$ Punkte, ermittelt mit dem CFT 1 (Weiss \& Osterland, 1980) und dem CFT 20 (Weiss, 1987), wobei die Spannweite 88 bis 
146 Punkte beträgt. Gemessen an der Normalverteilung der schulischen Intelligenz, in der $15 \%$ der Gesamtpopulation einen IQ von 120 und mehr Punkten besitzen, kann der hier eruierte Anteil von $24 \%$ als hoch bezeichnet werden. Der Grund für diese schiefe Verteilung liegt darin, dass sich die Stichprobe zur Hälfte aus FrühleserInnen und FrührechnerInnen zusammensetzt, die zu einem recht grossen Teil auch über hohe intellektuelle Fähigkeiten verfügen. 6.9\% dieser überdurchschnittlich Begabten sind der Gruppe der Underachiever zuzurechnen. Underachievement wird in unserer Studie definiert als IQ-Prozentrang $\geq 90$ und Schulleistungsprozentrang $\leq 50$ über mindestens drei Schuljahre. Overachiever sind in unserer Studie aufgrund der Fragestellung nicht erfasst worden.

\section{Untersuchungsdesign}

Im Mittelpunkt der Längsschnittstudie steht die Frage, welche Schulkarrieren und Entwicklungsverläufe junge Menschen zu verzeichnen haben, die bei Schuleintritt bereits lesen und rechnen können, d.h. welche Wirkungen vorschulisches Lesen- und Rechnenlernen hat, bezogen auf den Schulerfolg, die Berufswahl und die soziale Entwicklung. Dem Projekt liegt das Münchner Begabungsmodell (Heller, Mönks, Sternberg \& Subotnik, 2000) zugrunde, das von der Annahme angeborener Begabungsfaktoren ausgeht, die bei günstigen kognitiven und nicht-kognitiven Persönlichkeitsmerkmalen sowie günstigen Umgebungsfaktoren in herausragende Leistungen transformiert werden können. Entsprechend diesem Begabungsmodell wurden in fünf Untersuchungswellen Daten zu den relevanten Bereichen erhoben. (Mitte 1. Klasse: Ende 1995; Ende 1. Klasse: 1996; Mitte 3. Klasse: 1998; Mitte 5. Klasse: 2000; Mitte 8. Klasse: 2003). Anlässlich der Untersuchung im Jahr 2000 wurden die Bildungsaspirationen der Eltern eruiert. Im Jahr 2003 wurden die Jugendlichen selbst zu ihren eigenen beruflichen Ambitionen befragt. Die Befragungen fanden während des regulären Klassenunterrichts und mit der ganzen Klasse statt.

\section{Erhebungsinstrumente}

Das Instrumentarium soll einerseits das Ausmass widerspiegeln, in dem sich die Eltern um die berufliche Laufbahn ihrer Söhne und Töchter bemühen und andererseits das Ausmass der Übereinstimmung in ihren Bildungserwartungen insgesamt aufzeigen. Entsprechende Überlegungen finden sich in der Taxonomie von Trudewind (1975) und der von Sauer \& Gamsjäger (1996) diskutierten Weiterentwicklung. Nachfolgend werden die auf dieser Basis zusammengestellten Skalen erläutert.

Anregungsdimension des Elternhauses: Vier Items vom Typ: «Wir unterstützen unser Kind regelmässig bei den Hausaufgaben.» "Unser Kind besucht ausserhalb der Schule zusätzliche Angebote, die wir selbst finanzieren (Nachhilfe, begabungsfördernde Massnahmen etc.).» Die Antwortvorgaben waren viergestuft mit den Alternative $1=$ trifft überhaupt nicht zu bis $4=$ trifft sehr zu. $(\alpha=.65)$ 
Anspruchdimension des Elternhauses (,Bildungsaspirationen'): Vier Items vom Typ: «Unser Sohn soll als beruflichen Abschluss mindestens [Berufslehre, Fachschule, Handelsschule, Meister-/Technikerausbildung, Fachhochschule, universitäre Hochschule] erreichen.» "Gute Schulleistungen unseres Kindes sind uns wichtig.» Auch hier standen vier Antwortvorgaben zur Verfügung, die von $1=$ trifft überhaupt nicht zu bis $4=$ trifft sehr zu reichten. $(\square=.65)$

Anspruchdimension der Jugendlichen (,Berufsambitionen'): Drei Items vom Typ: «Ich möchte gerne folgenden Ausbildungsabschluss erwerben: Hochschule, Fachhochschule, Berufslehre.» "Für mich ist dieser Ausbildungsabschluss sehr wichtig." Auch hier standen vier Antwortvorgaben zur Verfügung, die von $1=$ trifft überhaupt nicht zu bis $4=$ trifft sehr zu reichten. $(\alpha=.71)$

Intellektuelle Fähigkeiten: Zur Erfassung der intellektuellen Fähigkeiten und zur Kontrolle von Intelligenzunterschieden wurde mit dem CFT 1 und dem CFT 20 (Weiss \& Osterland 1980; Weiss 1987) ein kulturfaires Verfahren herangezogen $(\alpha=.65$ resp. $\alpha=.86)$. Der CFT 20 beinhaltet auch die beiden schulnahen Ergänzungstests mit Wortschatz- und Zahlenfolgenaufgaben, welche verbale und numerische Elemente des Faktors Verarbeitungskapazität erfassbar machen.

Kreativität: Die Kreativität wurde mit dem TSD-Z von Urban \& Jellen (1994) gemessen $(\alpha=.89)$, der neben Aspekten des divergenten Denkens auch qualitative kreativitätsrelevante Komponenten berücksichtigt, zeichnerischen Fähigkeiten jedoch keine besondere Rolle zuweist.

Schulleistungen: In allen fünf Erhebungswellen wurden die Schulnoten über die Lehrpersonen erhoben, indem von den Fächern Deutsch und Mathematik jeweils nach der letzten Zeugnisnote gefragt wurde, die auf einer vierstufigen Ratingskala einzutragen war. Um Urteilstendenzen der Lehrpersonen in Form von Strenge- oder Mildeurteilen ausser Betracht zu lassen, wurden die beiden Noten innerhalb der Klasse standardisiert und zu einem Gesamtwert verdichtet. Ebenso wurde die aktuelle Leistungspositionierung des Schülers/der Schülerin in Deutsch und Mathematik erfragt (Klassenspitze; vorderes Leistungsdrittel, Mittelfeld, hinteres Leistungsdrittel).

Berufsstatus der Eltern: Aus den Verfahren von Bauer (1972) wurde die Kategorisierung der Schul- und Bildungsabschlüsse für den Berufsstatus der Eltern übernommen, allerdings schliesslich zu lediglich drei Kategorien verdichtet. Für Mutter und Vater wurde der höchste erreichte schulische und berufliche Abschluss jeweils getrennt kategorisiert. Aufgrund der hohen Korrelationen der Werte ( $\mathrm{r}=.61)$ wurde ein gemeinsamer Kennwert gebildet. 


\section{Ergebnisse}

Die statistischen Auswertungen gliedern sich in zwei Hauptabschnitte. Nach der Darstellung der Unterschiede zwischen Untersuchungs- und Vergleichsgruppe in drei verschiedenen Merkmalsbereichen werden im ersten Teil die Ausprägungen der Bildungserwartungen der Eltern dargestellt. Dabei wird untersucht, ob sich die erwarteten Zusammenhänge zwischen ihnen, den sozialstrukturellen Merkmalen und den CFT-Werten empirisch stützen lassen. Um die Elemente insgesamt zu betrachten und um mehrdimensionale Zusammenhänge herauszuarbeiten, werden in einem zweiten Schritt die Ergebnisse einer Clusteranalyse vorgestellt. Der dritte Schritt fokussiert schliesslich die Leistungsverläufe der Jugendlichen in den Clustern und diskutiert sie anhand weiterer Merkmale.

\section{Gruppenunterschiede}

Zunächst wird untersucht, inwiefern sich die erwarteten Unterschiede zwischen Untersuchungs- und Vergleichsgruppe in den Variablen abbilden. Es ergeben sich die in Tabelle 2 dargestellten Mittelwerte und Differenzen. Die Analyse zeigt, dass sich die Untersuchungs- von der Vergleichsgruppe nach acht Schuljahren noch in sechs Bereichen signifikant unterscheidet: in den intellektuellen Fähigkeiten, im Persönlichkeitsprofil, in der Leistungssituierung, in den Deutsch- und Mathematikleistungen sowie im Bildungsstatus der Eltern. Keine Unterschiede ergeben sich hinsichtlich der Kreativität sowie aller nicht das Persönlichkeitsprofil betreffenden Persönlichkeitsmerkmale (soziale Integration, Schulzufriedenheit, fachspezifische Interessen). Aufgrund dieser Unterschiede ist davon auszugehen, dass sie einen Einflussfaktor bei der Gruppenbildung im Rahmen der Clusteranalyse darstellen.

Tabelle 2: Gruppenunterschiede Ende achter Klasse (2003) ( ${ }^{\circ}:$ max. $=1$, min. $=4$; ${ }^{\circ}$ : $\max .1 ; \min .3 ; N=366$ )

\begin{tabular}{|l|l|c|c|c|}
\hline Merkmalsbereich & Kriterium & UG & VG & t-Test \\
\hline \multirow{3}{*}{ Begabungsfaktoren } & Intelligenz (CFT-20 Skala 2) & 120.2 & 109.1 & ${ }^{* *}$ \\
\cline { 2 - 5 } & Kreativität (TSD-Z) $^{*}$ Persönlichkeitsmerkmale & 66.7 & 65.3 & n.s. \\
\hline & Persönlichkeitsprofil & \\
\cline { 2 - 5 } & soziale Integration $^{\circ}$ & 2.56 & 2.83 & ${ }^{*}$ \\
\cline { 2 - 5 } & Schulzufriedenheit $^{\circ}$ & 2.40 & 2.38 & n.s. \\
\cline { 2 - 5 } & fachspezifische Interessen $^{\circ}$ & 2.45 & 2.65 & n.s. \\
\hline \multirow{3}{*}{ Leistungen } & Leistungssituierung $^{\circ}$ & 2.34 & 2.66 & ${ }^{* *}$ \\
\cline { 2 - 5 } & Deutsch $^{\circ}$ & 2.13 & 2.40 & ${ }^{*}$ \\
\cline { 2 - 5 } & Mathematik $^{\circ}$ & 2.21 & 2.48 & ${ }^{*}$ \\
\hline sozioökonomische Daten & Bildungsstatus der Eltern $^{\circ}$ & 2.03 & 1.84 & ${ }^{*}$ \\
\hline
\end{tabular}

${ }^{*} \mathrm{p}<.05 ;{ }^{* *} \mathrm{p}<.01$; n.s.=nicht signifikant $<\mathrm{UG}=$ Untersuchungsgruppe; $\mathrm{VG}=$ Vergleichsgruppe 


\section{Die Bildungsaspirationen der Eltern}

Die Ausprägung der Bildungsaspirationen der Eltern und der Berufsambitionen ihrer Kinder, differenziert nach kognitiven Fähigkeiten, Geschlecht und soziale Herkunft, sind in den Tabellen 3 und 4 dargestellt. Zunächst ist festzustellen, dass - bei einem durchschnittlichen Mittelwert von 2.5 - die Ausprägung der elterlichen Bildungsaspiration in beiden Dimensionen durchwegs als hoch zu bezeichnen ist und die beruflichen Ambitionen ihrer Söhne und Töchter übertrifft. Allerdings ist die Anspruchsorientierung nicht nur deutlich ausgeprägter als die Anregungsorientierung, sie fördert auch deutlichere Subgruppenunterschiede zutage. Eltern überdurchschnittlich begabter Schülerinnen und Schüler haben die höchsten Erwartungen, wenn es um den zukünftigen Bildungsabschluss ihres Kindes geht. Gleiches gilt für die Differenzierung nach Geschlecht. Ginge es nach dem Willen der Eltern, so sollten die Töchter deutlich seltener (MW=2.72) einmal eine weiterführende Schule besuchen oder eine höhere Bildungslaufbahn einschlagen als die Söhne (MW=2.91). Erwartungsgemäss ergeben sich auch signifikante Unterschiede hinsichtlich des Berufsstatus der Eltern: Akademisch geprägte Elternhäuser zeigen den höchsten Bildungsehrgeiz, doch ist zu beachten, dass die Erwartungsorientierungen in allen drei Statusniveaus überdurchschnittlich hoch sind. Mit Blick auf die Anregungsorientierung ergibt sich folgender Sachverhalt: Private Unterstützung und Förderung erhalten überdurchschnittlich begabte Kinder zwar deutlich häufiger als durchschnittlich begabte, doch ergeben sich zwischen Jungen und Mädchen oder in Bezug auf die berufliche Situierung der Eltern nur zufällige Differenzen. Gesamthaft gesehen lassen diese Befunde somit die Aussage zu, dass sich Bildungsaspirationen vor allem in Anspruchsorientierungen artikulieren, in Bezug auf den Anregungsgehalt jedoch am deutlichsten schichtspezifisch variieren.

Tabelle 3: Bildungsaspirationen der Eltern (erfasst im Jahr 2000), differenziert nach kognitiven Fähigkeitsprofilen, Geschlecht der Kinder und sozialer Herkunft)

\begin{tabular}{|l|c|c|c|c|}
\hline \multirow{2}{*}{ Population } & \multicolumn{2}{|c|}{$\begin{array}{c}\text { Anregungs- } \\
\text { orientierung }\end{array}$} & \multicolumn{2}{|c|}{$\begin{array}{c}\text { Anspruchs- } \\
\text { orientierung }\end{array}$} \\
\cline { 2 - 5 } & MW & $S$ & MW & S \\
\hline gesamt $(n=366)$ & 2.63 & 0.60 & 2.80 & 0.56 \\
\hline
\end{tabular}

\begin{tabular}{|l|l|l|l|l|}
\hline $\mathrm{IQ} \geq 120(\mathrm{n}=99)$ & 2.82 & 0.71 & 2.82 & 0.66 \\
\hline $\mathrm{IQ}<120(\mathrm{n}=267)$ & 2.51 & 0.42 & 2.79 & 0.46 \\
\hline statistische Signifikanz & \multicolumn{2}{|c|}{$*$} & \multicolumn{2}{|c|}{ n.s. } \\
\hline
\end{tabular}

\begin{tabular}{|l|l|l|l|l|}
\hline Jungen $(\mathrm{N}=179)$ & 2.72 & 0.73 & 2.91 & 0.67 \\
\hline Mädchen (N=187) & 2.65 & 0.52 & 2.72 & 0.46 \\
\hline statistische Signifikanz & \multicolumn{2}{|c|}{ n.s. } \\
\hline
\end{tabular}




\begin{tabular}{|l|c|c|c|c|}
\hline Arbeitermilieu & 2.53 & 0.61 & 2.81 & 0.61 \\
\hline kaufmännisch-gewerbliches Milieu (N=82) & 2.97 & 0.72 & 2.92 & 0.73 \\
\hline akademisches Milieu (N=172) & 2.85 & 0.53 & 3.00 & 0.54 \\
\hline statistische Signifikanz (N=112) & \multicolumn{2}{|c|}{$* *$} \\
\hline
\end{tabular}

Vierstufige Antwortvorgabe von 1=min. bis 4=max.; dreistufige Antwortvorgabe von 1=min. bis $3=\max$. Wertebereiche der Items: Anregungsorientierung (1-4); Anspruchsorientierung (1-4); Berufsstatus der Eltern (1=Arbeitermilieu, 2=kaufmännisch-gewerbliches Milieu; 3=akademisches Milieu); Mittelwerte (MW) und Standardabweichungen (S)

Signifikanzangaben: t-Test bzw. ${ }^{2-}$ Test; ${ }^{*}=\mathrm{p}<.05 ;{ }^{* *}=\mathrm{p}<.01 ; \mathrm{n} . \mathrm{s} .=$ nicht signifikant

Ein Blick in Tabelle 4 gibt Auskunft über die drei Jahre später erfassten Berufsambitionen der Jugendlichen. In der Tendenz fallen sie ähnlich aus wie die Bildungsaspirationen ihrer Eltern. Zwar zeigen sich erwartungsgemäss zwischen Jungen und Mädchen keine Unterschiede. Dass jedoch Gleiches für die kognitiven Fähigkeiten gilt, erstaunt, ebenso, dass die Unterschiede in Bezug auf die soziale Herkunft nicht deutlicher ausfallen. Möglicherweise wird damit eine immer wieder beschriebene Tendenz zur Selbstüberschätzung durchschnittlich begabter Jugendlicher manifest (vgl. Deutsche Shell, 2002, S. 64).

Tabelle 4: Bildungsambitionen der Jugendlichen (erfasst im Jahr 2003), differenziert nach kognitiven Fähigkeitsprofilen, Geschlecht und sozialer Herkunft

\begin{tabular}{|l|c|c|}
\hline Population & MW & S \\
\hline gesamt & 2.76 & 0.75 \\
\hline
\end{tabular}

\begin{tabular}{|l|l|l|}
\hline $\mathrm{IQ} \geq 120(\mathrm{n}=99)$ & 2.88 & 0.63 \\
\hline $\mathrm{IQ}<120(\mathrm{n}=267)$ & 2.70 & 0.82 \\
\hline statistische Signifikanz & \multicolumn{2}{|c|}{ n.s. } \\
\hline
\end{tabular}

\begin{tabular}{|l|l|l|}
\hline Jungen (N=179) & 2.74 & 0.81 \\
\hline Mädchen (N=187) & 2.80 & 0.62 \\
\hline statistische Signifikanz & \multicolumn{2}{|c|}{ n.s. } \\
\hline
\end{tabular}

\begin{tabular}{|l|c|c|}
\hline Arbeitermilieu & 2.56 & 0.61 \\
\hline kaufmännisch-gewerbliches Milieu (N=82) & 2.68 & 0.76 \\
\hline akademisches Milieu (N=172) & 2.89 & 0.60 \\
\hline statistische Signifikanz $(\mathrm{N}=112)$ & \multicolumn{2}{|c|}{$*$} \\
\hline
\end{tabular}

Vierstufige Antwortvorgabe von $1=\min$. bis 4=max.; dreistufige Antwortvorgabe von $1=\min$. bis 3=max. Wertebereiche der Items: Anregungsorientierung (1-4); Anspruchsorientierung (1-4); Berufsstatus der Eltern (1=Arbeitermilieu, 2=kaufmännisch-gewerbliches Milieu; 3=akademisches Milieu); Mittelwerte (MW) und Standardabweichungen (S)

Signifikanzangaben: t-Test bzw. ${ }^{2-}$ Test; ${ }^{*}=\mathrm{p}<.05 ;{ }^{* *}=\mathrm{p}<.01 ; \mathrm{n} . \mathrm{s} .=$ nicht signifikant 


\section{Typen von elterlichen Bildungsambitionen}

Um die bisher beschriebenen Elemente insgesamt zu betrachten und mehrdimensionale Zusammenhänge herauszuarbeiten, werden in einem nächsten Schritt Eltern und Kinder nach den Merkmalsausprägungen in den erhobenen Dimensionen beschrieben. Zu diesem Zweck wurde auf der Grundlage der Skalenwerte eine personenbezogene Clusteranalyse gerechnet, welche Eltern und Kinder nach dem Kriterium eines ähnlichen Antwortverhaltens in Gruppen einteilt. Unter inhaltlichen und formalen Kriterien erwies sich eine Lösung mit fünf Clustern als ideal. Die im Folgenden beschriebene Fünf-Cluster-Lösung klärt insgesamt 32.5\% der Gesamtvarianz auf den einbezogenen Variablen auf. In Abbildung 1 sind die fünf Gruppen von Eltern anhand der z-Werte dargestellt. Bereits ein flüchtiger Blick auf die einzelnen Typen lässt erkennen, dass Cluster 1 und 3 die stärksten Abweichungen aufweisen und das mit einander entgegengesetztem Profil. Cluster 2 und 4 unterscheiden sich in erster Linie in der Höhe der kognitiven Fähigkeiten, während Cluster 5 Profilausprägungen sowohl im negativen wie auch im positiven Bereich zeigt mit entsprechend unterschiedliche Profilkomponenten.

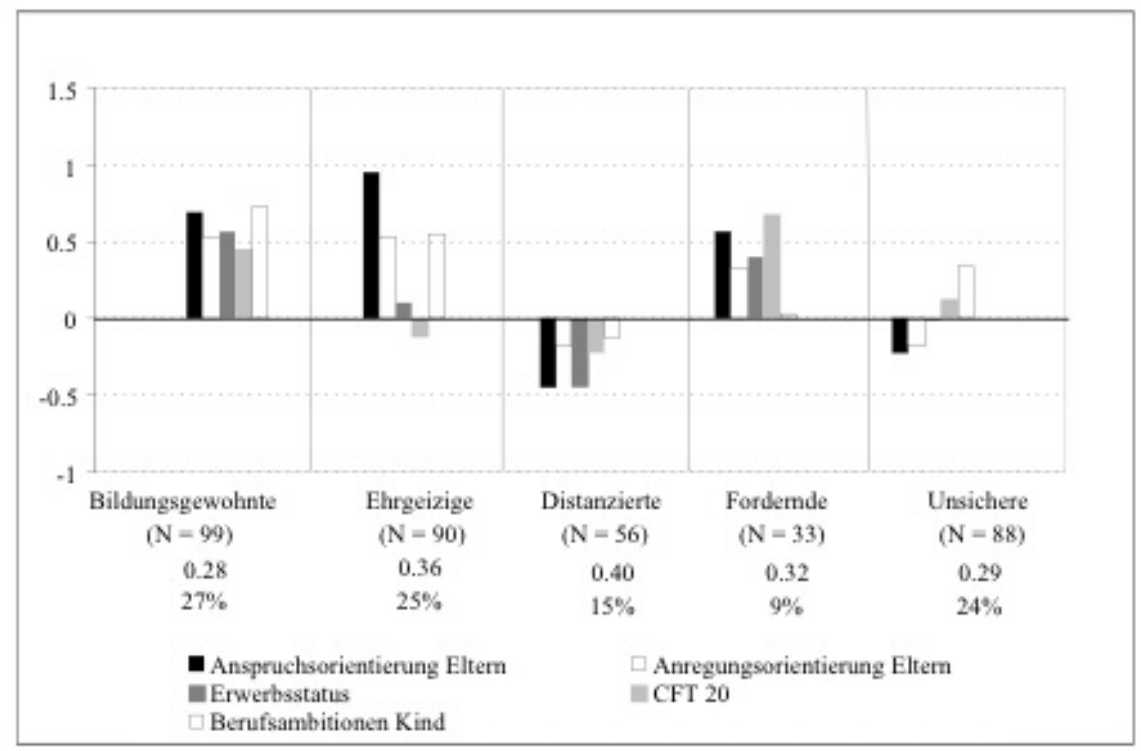

Abbildung 1: Cluster und z-Werte aus der Faktorenlösung mit fünf Clustern; (mit Anzahl, Konsistenzkoeffizient $\alpha$ und bzw. prozentualem Anteil der diesem Cluster zugeordneten Schülerinnen und Schüler)

Cluster I findet sich in 99 Fällen (27\% der Stichprobe). Eltern dieses Clusters sind vorwiegend in akademischen Berufen tätig und haben hohe implizite Er- 
wartungen an die Bildungslaufbahn ihres Kindes insofern, als dass sie gute Schulleistungen als selbstverständlich erachten. Deshalb wird das Cluster auch «Bildungsgewohnte» genannt. Das Anregungsmilieu ist vielfältig, aber keinesfalls ausschliesslich an der Schule orientiert. Davon zeugen die privat initiierten Fördermassnahmen, die vorwiegend auf den musischen, künstlerischen und sportlichen Bereich ausgerichtet sind. Die Kinder verfügen über hohe kognitive Fähigkeiten, und die Erwartungen der Eltern spiegeln sich in etwa in den beruflichen Ambitionen ihrer Söhne und Töchter. In Cluster II ( $n=90 ; 25 \%)$ sind die «ehrgeizigen Eltern» zusammengefasst, die vorwiegend dem kaufmännisch-gewerblichen Milieu angehören. Ihre hohen Bildungserwartungen spiegeln sich nicht nur in der Investition recht grosser finanzieller Mittel in private Fördermassnahmen, sondern auch in den Berufsambitionen ihrer Kinder. Angesichts ihrer lediglich durchschnittlichen kognitiven Profile müssen sie allerdings als eher überhöht bezeichnet werden. Cluster III ("Distanzierte») markiert in gewisser Hinsicht den Gegenpol zu Cluster II. Es beschreibt diejenigen 15\% $(n=56)$ der vorwiegend das Arbeitermilieu repräsentierenden Elternhäuser mit relativ geringen Bildungsambitionen und Interessen gegenüber der Schullaufbahn ihres Kindes. Auch die Anregungsaktivitäten fallen eher bescheiden aus. Die intellektuellen Fähigkeiten ihres Kindes sind lediglich durchschnittlich oder leicht unterdurchschnittlich, und seine beruflichen Ambitionen sind, den Eltern entsprechend, eher bescheiden. Cluster IV ( «Fordernde», 9\%; N=33) unterscheidet sich grundlegend von allen anderen Clustern. Die Eltern lassen sich zwar durch eine recht hohe Anspruchsorientierung kennzeichnen, halten sich jedoch mit privaten Anregungen und Fördermassnahmen eher zurück, was angesichts ihres vorwiegend mittelständisch-akademischen Erwerbsstatus und des höchsten kognitiven Profils ihres Kindes erstaunlich ist. Ebenso erstaunlich sind allerdings die Berufsambitionen dieser Jugendlichen, die sich nicht mit den Erwartungen der Eltern decken und gewissermassen einen Gegenpol darstellen. Cluster V umfasst die Gruppe "Unsichere» $(24 \% ; \mathrm{N}=88)$. Eltern aus kaufmännisch-gewerblichen und handwerklichen Berufen, in der Formulierung ihrer Bildungserwartungen als auch ihrer Anregungsaktivitäten deutlich zurückhaltend, bilden dieses Cluster und zwar deswegen, weil sie sich in der Anspruchs- und Anregungsdimension unsicher zeigen, sich jedoch für die Bildungslaufbahn ihres Kindes interessieren. Die Kinder weisen durchschnittliche kognitive Profile auf, zeigen sich jedoch ambitioniert, was ihre berufliche Zukunft anbelangt.

\section{Schullaufbabnen}

Welche Schullaufbahnen verbergen sich nun hinter diesen familiären Bildungsaspirationstypen? In Abbildung 2 ist die Leistungsentwicklung, über die fünf Messzeitpunkte dargestellt, operationalisiert als Mittelwerte der Noten in Deutsch und Mathematik. Als erstes fällt auf, dass die Schülergruppen mit unterschiedlichen Leistungsprofilen (Lese- und Mathematikvorkenntnisse) gestartet sind. Während die Schülerinnen und Schüler aus bildungsgewohnten El- 
ternhäusern stabil blieben und ihre Positionen bis zum Ende des achten Schuljahres halten konnten, zeichnet sich das Cluster der Fordernden durch eine deutliche Abwärtsmobilität nach der 5. Klasse aus. Ab diesem Zeitpunkt wurde es von den beiden Clustern der Unsicheren und der Ehrgeizigen überholt, die sich ihrerseits während der ganzen Grundschulzeit im Mittelfeld bewegten, jedoch ab der 5. Klasse zu Gunsten der Ehrgeizigen auseinanderdrifteten. Das Cluster der Distanzierten schliesslich ist im negativen Sinne immer stabil geblieben.



Abbildung 2: Schullaufbahnen und elterliche Bildungsambitionen ( $N=366)$

\section{Zusammenfassung und Diskussion der Ergebnisse}

Obwohl der Einfluss der Elternerwartungen hinsichtlich des Schulerfolgs ihrer Kinder allgemein als gross - zumindest grösser als der Einfluss der Schule - eingeschätzt wird, liegen bislang, von wenigen Untersuchungen abgesehen, kaum differenzierte Erkenntnisse vor über Umfang, Unterschiede und Zusammenhänge von Bildungsaspiration und Schullaufbahn. Aus diesem Grund wurde eine Studie zu diesem Themenbereich vorgestellt und dabei auch die intellektuellen Fähigkeiten einbezogen. Ausgangspunkt der theoretischen Überlegungen waren Aussagen zur Kapitaltheorie von Bourdieu, zu handlungstheoretischen Modellen und zur Selbstbestimmungstheorie. Sie legten das theoretische Gerüst für die Erklärung des elterlichen Einflusses direkter oder indirekter Art.

Wie ist auf diesem Hintergrund die Relevanz der Ergebnisse einzuschätzen und welche Begrenzungen der Studie sind zu beachten? Positiv ist vorerst festzu- 
halten, dass es im Rahmen dieser Längsschnittstudie gelungen ist, fünf Grundmuster von elterlichen Bildungserwartungen im Zusammenhang mit der Schullaufbahn des Sohnes oder der Tochter zu identifizieren. Zwei dieser Cluster - das der Bildungsgewohnten und der Fordernden - erweisen sich als bedeutsam hinsichtlich der Differenzierung nach Begabungsprofilen. Überdurchschnittlich begabte Jugendliche wachsen mehrheitlich in Familien mit ausgesprochen hohen Bildungsambitionen auf, die dann mit Schulerfolg verbunden sind, wenn die Anforderungsorientierung eher unauffällig, die Anregungsaktivitäten jedoch hoch sind. Damit lassen sich teilweise die Befunde aus der Hochbegabungsforschung bestätigen, dass sich Underachiever-Schullaufbahnen durch die Kombination von hoher Anspruchs- und bescheidener Anregungsorientierung definieren lassen, während sich Achiever-Karrieren durch eine niedrige Anspruchs- und eine hohe Anregungsdimension auszeichnen. Über das Verhältnis von Bedingung und Wirkung ist damit allerdings noch nichts ausgesagt, und es sei auf die Problematik der Underachievement-Forschung verwiesen, die bislang die Frage der Richtung nicht geklärt hat, meist jedoch davon ausgeht, Underachievement sei die Ursache mangelnder Leistungsmotivation. Nicht berücksichtigt bleibt damit auch das handlungstheoretische Postulat, wonach jeder Beeinflussung durch das Elternhaus eine Initiative der Heranwachsenden wie auch des näheren sozialen Umfelds gegenübersteht oder vorausgeht. So gesehen könnten hohe habitualisierte Anforderungsmuster von Eltern hoch begabter Kinder auch eine Antwort auf ihre Performanzeinbrüche darstellen. Im Gegensatz dazu erinnert das dritte identifizierte Cluster der Ehrgeizigen in verschiedener Hinsicht an das in der Literatur häufig beschriebene Konzept des Overachievements (Flammer \& Keller, 1978). Auch hier liegen relativ hohe elterliche Bildungsambitionen vor, allerdings mit dem Unterschied, dass sich die lediglich durchschnittlichen bis unterdurchschnittlichen kognitiven Profile in einer relativ günstigen Notenentwicklung und ausgeprägten jugendlichen Berufsambitionen abbilden. Über bisherige Erkenntnisse hinaus geht hingegen das fünfte Cluster der Unsicheren. Denn anders als erwartet finden sich als Pendant marginaler elterlicher Bildungserwartungen ausgeprägte Berufsambitionen des Kindes, und dies vor dem Hintergrund sowohl durchschnittlicher Intelligenz als auch im Wesentlichen problemlos verlaufender Schulkarrieren. Zumindest stützen solche Befunde die eingangs erörterten theoretischen Konzepte kaum. Möglich wäre allerdings die Erklärung, dass die Eltern zwar sehr zurückhaltende und von Unsicherheit geprägte Bildungserwartungen hegen, die jedoch die Förderung der Grundbedürfnisse des Kindes nicht tangieren, so dass intrinsische Bildungsmotivation entstehen kann.

Die Gesamtergebnisse replizieren die bereits bekannten Befunde, gehen jedoch in Teilbereichen darüber hinaus und differenzieren damit die eingangs dargelSSegten theoretischen Überlegungen. Insgesamt ist eine allgemein hohe Bildungsorientierung feststellbar, doch findet sich keine empirische Evidenz für die Annahme, Bildungsaspiration sei per se mit sozialer Schicht gekoppelt. Erst ihre 
Differenzierung in Teilaspekte verdeutlicht, dass sie sich vor allem als durchgehend hohe Anspruchsorientierung artikuliert und in Bezug auf den Anregungsgehalt, d.h. die privaten Stütz- und Fördermassnahmen, am deutlichsten schichtspezifisch variiert. Damit verbunden ist ein zweiter Befund, der die bisherigen Erkenntnisse erweitert. Wenn in erster Linie das Ausmass an privaten Anregungen und Unterstützungen und weniger der Ausbildungshintergrund ausschlaggebend ist, dann bleibt zu fragen, inwiefern dieser Fakt der Frage des ökonomischen und damit des kulturellen Kapitals eine neue Bedeutung verleiht. In Anlehnung an Bourdieu liesse sich argumentieren, dass Familien mit hohem ökonomischem Kapital schulnahe Freizeitangebote schneller ,einkaufen' und damit ihre Ambitionen über die stimulierenden intellektuellen Einflüsse Dritter auf ihr Kind einwirken lassen können. Diese alternative Möglichkeit der Aneignung von Bildungskapital macht jedoch eine neue, weil indirekte Zementierung der Schicht- und Bildungsabhängigkeit denkbar. Damit ist ein zweiter, allerdings widersprüchlicher Befund verknüpft. Er bezieht sich auf das Fehlen einer durchgehend engen Beziehung zwischen Bildungsaspiration der Eltern und Berufsambition ihrer Töchter und Söhne. Im traditionellen Verständnis, das von uni-direktiven Einflüssen der Eltern auf das Kind ausgeht, sind niedrige Bildungsaspirationen der Eltern für niedrige Berufsambitionen ihrer Kinder verantwortlich, wie dies im Cluster der Distanzierten der Fall ist. Geht man jedoch von der Reziprozität der Beziehungen aus und lässt man die Wirkrichtungen offen, dann können sie auch als enttäuschte Elternreaktion auf fehlende berufliche Perspektiven ihres Nachwuchses interpretiert werden und, wie dies in der Gruppe der Fordernden der Fall ist, in besonders hohe Ansprüche münden. Möglich ist jedoch auch das Erklärungsmuster, dass Schul- und Ausbildungswünsche der Eltern durch die zunehmende Bedeutung der Peer-Umwelten relativiert und mit Gegenentwürfen beantwortet werden. Als Folie über die in dieser Untersuchung eruierte Kombination ,niedrige Bildungsaspiration der Eltern und hohe Berufsambitionen des Kindes' gelegt, ergibt sich ein doppeltes Erklärungsmuster: Demnach könnten (a) zurückhaltende bis unsichere Elternerwartungen die Leistungsbereitschaft und die beruflichen Ambitionen des Kindes geradezu herausfordern oder (b) die gute Leistungsentwicklung - möglicherweise in einem stimulierenden Peerkontext - Elternambitionen geradezu überflüssig machen.

Insgesamt bilden diese Typen fünf Schemata, die sich als Grammatik familialer Bildungsambition begreifen lassen. Sie machen klar, dass Leistungen von Schülerinnen und Schülern auf spezifische Weise von den Lernbedingungen in der Familie abhängen. Auf der Folie der Eingangsfrage - inwiefern Eltern als Erfolgspromotoren ihrer Söhne und Töchter wirken - lenken solche Einflussgrössen auf die Frage der Schulqualität. Solche Abhängigkeiten sollte die Qualitätsdiskussion aufnehmen und das pädagogische Handeln und Unterlassen der Eltern berücksichtigen.

Schliesslich stellt sich noch die Frage nach der Begrenzung des Aussagengehalts der Studie, die insgesamt vier Hinweise notwendig macht. Erstens ist da- 
von auszugehen, dass die Spezifik unserer Stichprobe - fast ein Viertel der Probandinnen und Probanden verfügen über überdurchschnittliche kognitive Fähigkeiten - eine Generalisierung der Befunde verbietet. Zweitens ist es durchaus möglich, dass es sich bei den gefundenen fünf Grundtypen elterlicher und jugendlicher Bildungserwartungen um wandelbare Einstellungs- und Handlungsmuster handelt, die sich vor allem in Zeiten von Stabilität oder Instabilität, insbesondere von Statuspassagen, ändern. Eltern stellen drittens selbstverständlich nicht die gesamte bildungs- und sozialisationsrelevante Umwelt dar. Welche sozialen Umwelt-Konfigurationen zusätzlich eine Rolle spielen, kann aus den vorliegenden Daten nicht abgelesen werden, weil weder die Bedeutung der Peeroder anderer Freizeit-Umwelten, der Interaktions- und Strukturbedingungen der schulischen Umwelt noch der weiteren innerfamiliären Beziehungen (Geschwister, Verwandtschaft) erhoben worden sind. Viertens gilt zu beachten, dass die Ergebnisse offen lassen müssen, ob unsere Kausalannahmen und Wirkvermutungen gerechtfertigt sind, respektive wie Übereinstimmungen oder Abweichungen zwischen den Aspirationen der Eltern und den Ambitionen ihres Kindes zustande gekommen sind. Eine solche Überprüfung soll in der nächsten Erhebungsrunde im Jahr 2006 im Rahmen einer biographische Analyse, die die sozialen Bedingungen in Kindheit und Jugend untersucht, erfolgen. Aus solchen Begrenzungen ergeben sich somit auch Impulse für neue Fragestellungen: Wenn es zutrifft, dass es weniger zentral ist, welchen Ausbildungshintergrund die Eltern haben als wie sie sich dem Kind gegenüber verhalten, dann interessiert, wie diese Aspirationen und Ambitionen beim weiteren «Weben dieses Kleids der Lebenstüchtigkeit» (Fend, 1998, S. 32) zukünftig eingearbeitet werden.

\section{Literatur}

Allen, J., Moore, C.M., Kuperminc, G. \& Bell, K. (1998). Attachment and psycho-socialfunctioning. Child Development, 69, 1406-1419.

Bauer A. (1972). Ein Verfahren zur Messung des für Bildungsverhalten relevanten sozialen Status (BRSS). Heidelberg: Springer.

Bierman, K.L. (1996). Family school links: An overview. In A. Booth \& J.F. Dunn (Eds.), Family school links (pp. 275-387). Mahaw: Lawrence Erlbaum.

Bloom, B. S. (1985). Generalizations about talent development. In B.S. Bloom (Ed.), Developing talent in young people (pp. 507-549). New York: Ballantine Books.

Bourdieu, P. (1983). Ökonomisches Kapital, kulturelles Kapital, soziales Kapital. In R. Kreckel (Hrsg.), Soziale Ungleichheiten (S. 183-198). Göttingen: Schwartz.

Bourdieu, P. (1992). Die feinen Unterschiede. Frankfurt a.M.: Suhrkamp.

Bronfenbrenner, U. (1981). Die Ökologie der menschlichen Entwicklung. Stuttgart: Enke.

Coleman, J.S. (1988). Social capital in the creation of human capital. American Journal of Sociology, 94, 95-120.

Coleman, J.S., Campbell E.Q., Hobson C.J., McPartland, J., Mood A.M., Weinfeld, F.D. \& York R.L. (1966). Equality and educational opportunity. Washington D.C.: United States Department of Health, Education, and Welfare. Office of Education.

Cooper, H., Lindsay, J.J., \& Nye, B. (2000). Homework in the home. How students, family, and parenting-style differences relate to the homework process. Contemporary Educational Psychology, 25, 464-487. 
Cornell, D.G. (1984). Families of gifted children. Ann Arbor, Michigan: UMI Research Press.

Csikszentmihalyi, M. \& Rathunde, K. (1998). The development of the person: An experiential perspective on the ontogenesis of psychological complexity. In W. Damon \& R.M. Lerner (Eds.), Theoretical models of human development: Vol. 1. Handbook of Child Psycho$\log y$ (pp. 635-684). New York: Wiley.

Dachverband Lehrerinnen und Lehrer Schweiz (LCH). (2004). Der LCH zur Elternmitwirkung auf Schulebene. Positionspapier. Online: [http://www.lch.ch/docs/presse/Position_Elternmitw.pdf] (2. Juni 2005).

Deci, E.L., Ryan, R.M. (1993). Die Selbstbestimmungstheorie der Motivation und ihre Bedeutung für die Pädagogik. Zeitschrift für Pädagogik, 2, 223-238.

Deutsche Shell (Hrsg.), Jugend 2002. Zwischen pragmatischem Realismus und robustem Materialismus. Frankfurt: Fischer.

Fend, H. (1998). Eltern und Freunde. Soziale Entwicklung im Jugendalter. Bern: Huber.

Flammer, A., \& Keller, B. (1978). Overachievement und underachievement. In K.J. Klauer (Hrsg.), Handbuch der Pädagogischen Diagnostik, Band 2 (S. 1037-1044). Düsseldorf: Schwann.

Fraser, B.J., Walberg, H.J. Welch, W.W., \& Hattie, J.A. (1987). Synthesis of educational productivity research. International Journal of Educational Research, 11, 2, 145-252.

Georg, W. \& Zinnecker, J. (1996). Philosophien des Statuserwerbs: Ihre Weitergabe von der Eltern- auf die Kindergeneration. In J. Zinnecker \& R.K. Silbereisen (Hrsg.). Kindheit in deutschland. Aktueller Survey über Kinder und ihre Eltern (S. 137-143). Weinheim: Juventa.

Hampsch, H. (1998). Freizeit und Schule. Die Selektion von Freizeitaktivitäten und ihr Einfluss auf schulisches Leistungs- und Sozialverhalten. Frankfurt a.M.: Lang.

Heller, K.A., Mönks, F.J., Sternberg, R.J. \& Subotnik, R.F. (Eds.), (2000). International handbook of giftedness and talent. Amsterdam: Elsevier.

Helmke, A., Schrader, F.-W. \& Lehneis-Klepper, G. (1991). Zur Rolle des Elternverhaltens für die Schulleistungsentwicklung ihrer Kinder. Zeitschrift für Entwicklungspsychologie und Pädagogische Psychologie, 23, 1-22.

Holodynski, M. \& Oerter, R. (2002). Motivation, Emotion und Handlungsregulierung. In R. Oerter \& L. Montada (Hrsg.), Entwicklungspsychologie (S. 551-589). Weinheim: Beltz.

Kramer, W. \& Werner, D. (1998). Familiäre Nachhilfe und bezahlter Nachhilfeunterricht. Köln: Institut der Deutschen Wirtschaft.

Krapp, A. (1992). Interesse, Lernen und Leistung. Neuere Forschungsansätze in der Pädagogischen Psychologie. Zeitschrift für Pädagogik, 38, S. 747-770.

Krumm, V. (1996). Schulleistung - auch eine Leistung der Eltern. Die heimliche und die offene Zusammenarbeit von Eltern und Lehrern und wie sie verbessert werden kann. In W. Specht \& J. Thonhauser (Hrsg.), Schulqualität: Entwicklungen - Befunde - Perspektiven (S. 256-290). Innsbruck-Wien: StudienVerlag.

Marjoribanks, K. (1979). Families and their learning environments: An empirical analysis. London: Routledge and Kegan Paul.

McCall, R.B., Evahn, C. \& Kratzer, L. (1992). High school underachievers. Newbury Park, CA: Sage.

Miller, A. (1980). Das Drama des begabten Kindes und die Suche nach dem wahren Selbst. Frankfurt: Suhrkamp.

Moser, H. (1992). Zum Verhältnis von Familie, Freizeit und Schule. Basel: Reihe FHS.BB, Nr. 1.

Neuenschwander, M.P. (2004). Konzepte und Forschungsbefunde zur Eltern-Lehrer-Zusammenarbeit. In Schweizerische Gesellschaft für Bildungsforschung (Hrsg.), Beiträge des Jahreskongresses Schule und Familie - Perspektiven einer Differenz (S. 1-19). Aarau: Schweizerische Koordinationsstelle für Bildungsforschung SKBF.

Neuenschwander, M.P., Balmer, T., Gasser, A. Golz, S., Hirt, U. et al. (2005). Schule und Familie - was sie zum Schulerfolg beitragen. Bern: Haupt. 
Pekrun, R. (1997). Kooperation zwischen Elternhaus und Schule. In L.A. Vaskovics \& H. Lipinski (Hrsg.), Familiale Lebenswelten und Bildungsarbeit (S. 51-79). Opladen: Leske \& Budrich.

Pekrun, R. (2001). Familie, Schule und Entwicklung. In S. Walper \& R. Pekrun (Hrsg.), Familie und Entwicklung. Aktuelle Perspektiven der Familienpsychologie (S. 84-105). Göttingen: Hogrefe.

Plowden, L. (Ed.), (1967). The Plowden Report: Children and their primary schools. London: HMSO.

Rolff, H.-G., Bauer, K.-O., Klemm, K. \& Pfeiffer, H. (Hrsg.). (1996). Jahrbuch der Schulentwicklung. Daten, Beispiele und Perspektiven (Bd. 9). Weinheim: Juventa.

Sauer, J. \& Gamsjäger, E. (1996). Ist Schulerfolg vorhersagbar? Die Determinanten der Grundschulleistung und ihr prognostischer Wert für den Sekundarschulerfolg. Göttingen: Hogrefe.

Stecher, L. (1999). Bildungsehrgeiz der Eltern, soziale Lage und Schulbesuch der Kinder. In R. Silbereisen \& J. Zinnecker (Hrsg), Entwicklung im sozialen Wandel (S. 337-356). Weinheim: Psychologie Verlags Union.

Stöckli, G. (1992). Schulische Übergänge und Emotionen in der Eltern-Kind-Beziehung. Psychologie in Erziehung und Unterricht, 39, 116-124.

Tettenborn, A. (1996). Familien mit hochbegabten Kindern. Münster: Waxmann.

Trudewind, C. \& Windels, A. (1991). Elterliche Einflussnahme auf die kindliche Kompetenzentwicklung: Schulleistungseffekte und ihre motivationale Vermittlung. In R. Pekrun \& H. Fend (Hrsg.), Schule und Persönlichkeitsentwicklung (S. 131-148). Stuttgart: Enke.

Trudewind, C. (1975). Häusliche Umwelt und Motiventwicklung. Göttingen: Hogrefe.

Ulich, K. (1989). Eltern und Schüler. Die Schule als Problem in der Familienerziehung. In Zeitschrift für Sozialisationsforschung und Erziehungssoziologie, 9, 179-194.

Urban, K.K., Jellen, H.G. (1994). Test zum Schöpferischen Denken - Zeichnerisch (TSD-Z) . Frankfurt: Swets.

Weiss, R. (1987). Grundintelligenztest CFT 20, Skala 2 mit Wortschatztest (WS) und Zablenfolgentest $(Z F)$. Göttingen: Hogrefe.

Weiss, R. \& Osterland, J. (1980). Grundintelligenztest CFT 1. Braunschweig: Westermann.

Whitmore, J. (1980). Giftedness, conflict and underachievement. US: Allyn and Bacon.

Wild, E. \& Hofer, M. (2002). Familien mit Schulkindern. In M. Hofer, E. Wild \& P. Noack (Hrsg.), Lehrbuch Familienbeziehungen (S. 216-240). Göttingen: Hogrefe.

Wild, E. (2001). Familiale und schulische Bedingungen der Lernmotivation von Schülern. Zeitschrift für Pädagogik, 47, 4, 481-499.

Zinnecker, J. \& Georg, W. (1996). Soziale Interaktion in der Familie und ihre Wirkung auf Schuleinstellung und Schulerfolg der Kinder. In J. Zinnecker \& R.K. Silbereisen (Hrsg.), Kindheit in Deutschland. Aktueller Survey über Kinder und ihre Eltern (S. 347-256). Weinheim: Juventa.

Schlagworte: Bildungsaspirationen, Berufsambitionen, Begabung, Schullaufbahn 


\title{
Aspiration à la formation, don et carrière scolaire : Les parents sont-ils les promoteurs du succès?
}

\begin{abstract}
Résumé
L'article étudie la corrélation entre les aspirations à la formation des parents et la carrière scolaire de leurs enfants. La base empirique est livrée par une étude longitudinale auprès de 400 jeunes âgés aujourd'hui de 17 ans portant sur les effets de l'acquisition de compétences en matière de lecture et de mathématique avant l'entrée à l'école. L'étude a cherché à savoir dans quelle mesure les aspirations à la formation des parents se retrouvent dans le succès scolaire de leurs enfants et dans leurs ambitions professionnelles à la fin de la période scolaire obligatoire, et si cela est particulièrement vrai pour des familles avec des enfants particulièrement doués. Fondamentalement, les analyses suggèrent une distinction entre cinq catégories fondamentales d'aspiration parentale à la formation, deux catégories apparaissant comme significatives eu égard à la différenciation selon des profils de don élevé: (a) un groupe de parents cultivés et ambitieux à cet égard dont les enfants ont suivi une carrière scolaire réussie et développé des ambitions professionnelles comparables; (b) un groupe de parents avec des tendances polaires, nommés «les exigeants", parce que leurs aspirations à la formation se manifestent surtout dans une exigence élevée, mais leurs enfants ont plutôt échoué dans leur carrière scolaire, et leurs ambitions professionnelles ne se reflètent pas dans les désirs des parents.
\end{abstract}

Mots clés: aspirations à la formation, ambitions professionnelles, don, carrière scolaire

\section{Aspirazione alla formazione, talenti e carriera scolastica: $i$ genitori quali "promotori di successo"?}

\section{Riassunto}

L'articolo studia la correlazione esistente tra le aspirazioni dei genitori e la carriera scolastica dei figli. La base di dati empirici è fornita da uno studio longitudinale su 400 giovani, oggi diciassettenni, incentrato sugli effetti dell'acquisizione di competenze in materia di lettura e matematica prima dell'entrata nella scuola. Lo studio ha cercato di mettere in evidenza in quale misura le aspirazioni dei genitori in materia di formazione sono riscontrabili nel successo scolastico dei loro figli e nelle loro ambizioni professionali alla fine della scuola dell'obbligo. Si è inoltre cercato di verificare se questa correlazione è particolarmente marcata nei casi di bambini molto dotati. Le analisi suggeriscono una suddivisione in 5 categorie di base in funzione delle aspirazioni alla formazione dei genitori. Due di queste categorie si rivelano essere significative in relazione alla differenziazione di profili di figli particolarmente dotati: a) un gruppo di genitori 
con una formazione elevata e molto ambiziosi i cui figli hanno successo nella loro carriera scolastica e sviluppano ambizioni professionali conseguenti e b) un gruppo di genitori che potremmo definire "gli esigenti", in quanto le loro aspirazioni alla formazione per i figli sono elevate, ma questi ultimi sono sovente confrontati con l'insuccesso scolastico e non maturano ambizioni professionali coerenti con i desideri dei genitori.

Parole chiave: aspirazioni rispetto alla formazione, ambizioni professionali, talento, carriera scolastica

\section{Educational aspiration, talent and schooling: Parents as promoters of success?}

\section{Summary}

The article examines the connection between the educational aspirations of parents and the path of schooling undertaken by their sons and daughters. Serving as empirical basis is a longitudinal study of 40017 -year-olds on the effects of the pre-school acquisition of competence in reading and mathematics. The analysis examines the extent to which the educational aspirations of parents constrain the scholarly success of their child and its professional ambitions at the end of the period of compulsory schooling; and whether this is particularly applicable for families with highly gifted children. Essentially, the analysis suggests a differentiation between five basic patterns of parental educational aspirations, whereby two patterns demonstrate significance with regard to a differentiation according to high talent profiles: (a) a group of parents that are "accustomed to education" and ambitious, whose child has successfully completed its course of schooling and has also developed similar professional ambitions to those of its parents; (b) a group of parents with diametrically opposed tendencies, designated «expectant parents", due to the fact that their educational aspirations are predominantly manifested in high demands, but where their child's school career is somewhat associated with failure and whose professional ambitions do not reflect the wishes of the parents.

Key words: educational aspirations, professional ambitions, talent, school career 
